\title{
Galaxies infalling into groups: filaments versus isotropic infall
}

\author{
Héctor J. Martínez, ${ }^{1 \star}$ Hernán Muriel ${ }^{1,2}$ and Valeria Coenda ${ }^{1,2}$ \\ ${ }^{1}$ Observatorio Astronómico, Universidad Nacional de Córdoba, Laprida 854, X5000BGR Córdoba, Argentina \\ ${ }^{2}$ Instituto de Astronomía Teórica y Experimental (IATE), CONICET-Universidad Nacional de Córdoba, Laprida 854, X5000BGR Córdoba, Argentina
}

Accepted 2015 October 1. Received 2015 September 8; in original form 2015 July 16

\begin{abstract}
We perform a comparative analysis of the properties of galaxies infalling into groups classifying them accordingly to whether they are: falling along filamentary structures; or they are falling isotropically. For this purpose, we identify filamentary structures connecting massive groups of galaxies in the SDSS. We perform a comparative analysis of some properties of galaxies in filaments, in the isotropic infall region, in the field, and in groups. We study the luminosity functions (LF) and the dependence of the specific star formation rate (SSFR) on stellar mass, galaxy type, and projected distance to the groups that define the filaments. We find that the LF of galaxies in filaments and in the isotropic infalling region are basically indistinguishable between them, with the possible exception of late-type galaxies. On the other hand, regardless of galaxy type, their LFs are clearly different from that of field or group galaxies. Both of them have characteristic absolute magnitudes and faint end slopes in between the field and group values. More significant differences between galaxies in filaments and in the isotropic infall region are observed when we analyse the SSFR. We find that galaxies in filaments have a systematically higher fraction of galaxies with low SSFR as a function of both, stellar mass and distance to the groups, indicating a stronger quenching of the star formation in the filaments compared to both, the isotropic infalling region, and the field. Our results suggest that some physical mechanisms that determine the differences observed between field galaxies and galaxies in systems, affect galaxies even when they are not yet within the systems.
\end{abstract}

Key words: galaxies: evolution-galaxies: groups: general-galaxies: luminosity function, mass function - galaxies: star formation-galaxies: statistics.

\section{INTRODUCTION}

The large-scale structure of the Universe is characterized by the presence of filaments which intersect at nodes wherein groups and clusters of galaxies are found (see Bond, Kofman \& Pogosyan 1996). Filaments are visually the most dominant structures in the distribution of galaxies and can be seen extending over scales up to tens of megaparsecs. The hierarchical models of structure formation predict that groups and clusters grow by the continuous accretion of galaxies. This accretion usually happens along filaments in a non-isotropic way (e.g. Ebeling, Barrett \& Donovan 2004).

Large galaxy redshift surveys such as the Sloan Digital Sky Survey (SDSS, York et al. 2000) as well as $N$-body simulations have motivated the implementation of several methods to identify filaments (e.g. Stoica, Martínez \& Saar 2010; Bond, Strauss \& Cen 2010; Aragón-Calvo, van de Weygaert \& Jones 2010). Many of the algorithms make use of the fact that filaments are the bridges that connect systems of galaxies (Pimbblet, Drinkwater \& Hawkrigg 2004; Colberg, Krughoff \& Connolly 2005; Pimbblet 2005; González \&
Padilla 2010; Smith et al. 2012; Zhang et al. 2013; Alpaslan et al. 2014). Colberg et al. (2005) suggested that the probability of finding a filament between systems of galaxies is strongly dependent on their separation. Zhang et al. (2013) used the SDSS Data Release 8 (Aihara et al. 2011) and detected filaments using a sample of more than 50000 clusters of galaxies. They selected cluster pairs separated by less than $35 h^{-1} \mathrm{Mpc}$ and found that richer clusters are connected to richer filaments.

Independently of the extension or the geometry, filaments are overdensities of galaxies and as such can affect the evolution of galaxies. The role of the filaments in this process has not been extensively studied. Zhang et al. (2013) studied the colour and luminosity distribution of galaxies in filaments. They found that filament galaxies are bluer and fainter than cluster members. Guo, Tempel \& Libeskind (2015) studied the satellite luminosity function of primary galaxies and found that the filamentary environment can increase by a factor of 2 the abundance of satellites compared with non-filament galaxies. They concluded that the filamentary environment may have a strong effect on the efficiency of galaxy formation.

The region connecting filaments with clusters of galaxies is the so called infall region that extends from the outskirts of a cluster up 
to several virial radii. Porter et al. (2008) found that galaxies falling into a cluster along filaments, are likely to undergo an enhancement of their star formation before they reach the virial radius of the cluster. Similarly, Mahajan, Raychaudhury \& Pimbblet (2012) reported an excess of star forming galaxies in the outskirts of dynamically unrelaxed clusters and associated this phenomenon to the infall of galaxies through straight filaments. They concluded that a relatively high galaxy density in the infalling regions of unrelaxed clusters produced momentary bursts of star formation.

Regardless of the filamentary structure, the cluster infall region has been extensively studied. Ellingson et al. (2001) studied the composite radial distributions of different stellar populations as a function of clustercentric radius. They found no evidence at any radius within the clusters for an excess of star formation over that seen in the field (see also Rines et al. 2005; Verdugo, Ziegler \& Gerken 2008). The general agreement is that galaxy properties converge to those of field galaxies at 2-3 virial radii. Group/filament pre-processing may play an important role in transforming galaxies before they enter into the cluster environment. It has also been suggested that a significant fraction of galaxies at large radii have passed through the core region of the cluster and have undergone environmental transformation within the virial radius (see Muriel \& Coenda 2014 and references therein). It has also been studied the properties of galaxies in the infalling region at intermediate/high redshifts. Just et al. (2015) found evidence of pre-processing of galaxies in the infall region of clusters in the redshift range $0.4<$ $z<0.8$. These authors found that at $z \sim 0.6$, the fraction of red galaxies in the infall region is larger than in the field (see also Moran et al. 2007 and Patel et al. 2011)

In this paper, we study the population of galaxies in the infalling region of massive groups taken from Zandivarez \& Martínez (2011). We distinguish between galaxies infalling into groups along filaments and those that are in the infalling region but outside filaments, which we refer to as isotropic infalling galaxies. We aim to characterize how these two infalling regions affect the star formation in galaxies. Based on the fact that filaments are the bridges that connect systems of galaxies, we first implement an algorithm to search for filaments connecting pairs of massive groups. Then, we stack the galaxy population around these groups into a sample of galaxies in filaments, and another of isotropically infalling galaxies. We compare these two populations with the galaxies in the groups that are connected by the filaments, and with a sample of field galaxies. This paper is organized as follows: we identify filaments using groups of galaxies and define our samples of galaxies in Section 2; we compare the properties of galaxies in filaments and those isotropically infalling into groups with field galaxies and galaxies in groups in Section 3; we discuss our results and present our conclusions in Section 4. Throughout this paper we use Petrosian magnitudes, in the $\mathrm{AB}$ system, and corrected for Galactic extinction using the maps by Schlegel, Finkbeiner \& Davis (1998). Absolute magnitudes and distances have been computed assuming a flat cosmological model with parameters $\Omega_{0}=0.3, \Omega_{\Lambda}=0.7$ and $H_{0}=100 h \mathrm{~km} \mathrm{~s}^{-1} \mathrm{Mpc}^{-1} . K$-corrections have been computed using the method of Blanton et al. (2003a, KCORRECT version 4.1). We have adopted a band shift to a redshift 0.1 for the $r$ band (hereafter ${ }^{0.1} r$ ), i.e. to approximately the mean redshift of the main galaxy sample of SDSS.

\section{THE SAMPLES}

The purpose of this paper is to study how the star formation of galaxies infalling into groups are differently affected by the envi- ronment, depending on whether they are in filaments or not. The samples of groups used in this paper were drawn from the sample of groups identified by Zandivarez \& Martínez (2011, hereafter ZM11) in the Main Galaxy Sample (Strauss et al. 2002) of the seventh data release of SDSS (Abazajian et al. 2009). They used a standard friends-of-friends algorithm (Huchra \& Geller 1982) to link galaxies into groups. The redshift-dependent linking length of the algorithm takes into account redshift space distortions. ZM11 implemented a complementary identification procedure using a higher density contrast in groups with at least 10 observed members, in order to split merged systems and clean up spurious member detection. The authors computed group virial masses from the virial radius of the systems and the velocity dispersion of member galaxies (Limber \& Mathews 1960; Beers, Flynn \& Gebhardt 1990). The catalogue of ZM11 comprises 15961 groups with more than four members, adding up to 103342 galaxies. We refer the reader to ZM11 and references therein for further details of group identification.

\subsection{Filaments connecting groups of galaxies}

A vast majority of the studies in the literature related to filaments, have focused the attention on filaments connecting clusters of galaxies. Since our aim is not the creation of a complete catalogue of filaments connecting groups, we restrict our analysis to massive groups at this point. Arguably, this choice gives us better chances of finding actual overdensities of galaxies stretching between systems. From the ZM11 catalogue we select all groups with virial mass above the catalogue's median mass $\left(\log \left(M_{\text {vir }} / h^{-1} \mathrm{M}_{\odot}\right) \geq 13.5\right)$ and in the redshift range $0.05 \leq z \leq 0.15$. We use this subset to identify pairs $(1,2)$ of groups defined by the following criteria: (1) the difference of the radial velocities of their baricentres $\left(\Delta V_{12}\right)$ is less than a chosen value $\left.\Delta V_{\max },\left|\Delta V_{12}\right| \leq \Delta V_{\max } ; 2\right)$ the projected distance between their baricentres $\left(\Delta_{12}\right)$ is smaller than a given value $\Delta_{\max }$ while being two clearly separated groups in the sky, i.e. they are separated by a projected distance larger than the sum of their projected virial radii: $r_{\mathrm{pv}}^{(1)}+r_{\mathrm{pv}}^{(2)} \leq \Delta_{12} \leq \Delta_{\max }$. We choose $\Delta_{\max }=$ $10 \mathrm{~h}^{-1} \mathrm{Mpc}$ and $\Delta V_{\max }=1000 \mathrm{~km} \mathrm{~s}^{-1}$. According to Zandivarez, Merchán \& Padilla (2003), groups in the mass range under consideration here have redshift space correlation length $s_{0} \sim 11 h^{-1} \mathrm{Mpc}$, thus, for the purposes of our work, we do not search for inter-group filaments spanning larger redshift space distances. We call nodes to groups that are part of a pair according to the conditions (1) and (2). Nodes can be part of more than one pair.

Since filaments are overdense zones compared to the mean galaxy number density, we select group pairs that are linked by overdensities in the galaxy distribution. We use all DR7 MGS galaxies in the redshift range under consideration and with apparent magnitudes $14.5 \leq r \leq 17.77$ and proceed as follows:

(i) First, we clean up the MGS of all galaxies contained in cylinders centred on groups and oriented along the line of sight with dimensions that escalate with group size. We find that we can exclude all galaxies in ZM11 groups if we choose the cylinders to have projected radius $1.7 r_{\mathrm{pv}}$ and height $4.3 \sigma$, where $r_{\mathrm{pv}}$ and $\sigma$ are the projected virial radius and the velocity dispersion of the group, respectively. This also excludes other galaxies that are geometrically close to the groups in redshift space, thus ours is a more conservative choice than only discarding galaxies in groups.

(ii) We define a Cartesian coordinate system whose origin is located in the geometric centre $(O)$ of each group pair with the $x$-axis oriented along the line connecting the centres of the groups, the $y$-axis orthogonal to the former in the plane of the sky, and the 


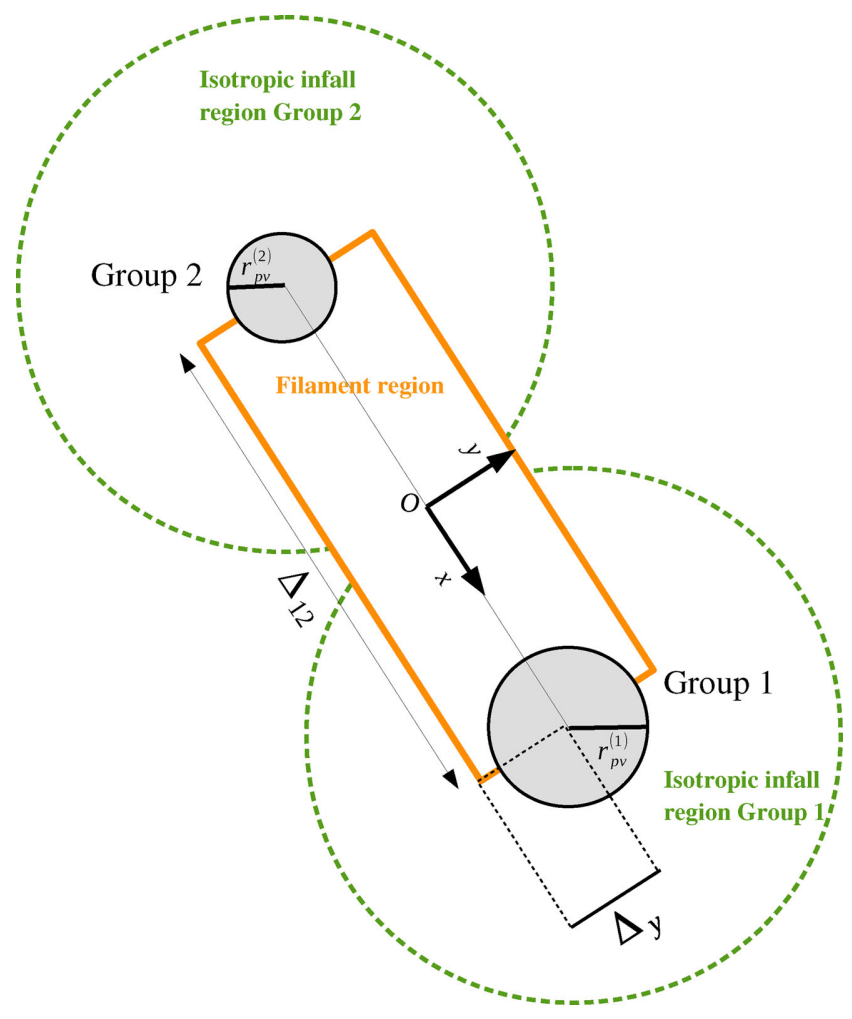

Figure 1. A plane of the sky projection of two hypothetical galaxy groups (grey circles) and the geometry we use to define the infalling regions: green dashed circles enclose the isotropic infall regions, and the orange rectangle defines the filament region. See text for details.

$z$-axis pointing outwards along the line of sight, see Fig. 1. Hereafter, to avoid confusion with the letter we use to denote redshift, $z$, we will refer to the line-of-sight axis as the $v$-axis. We consider the filament region to be a rectangular cuboid in redshift space defined by: $|x| \leq \Delta_{12} / 2,|y| \leq \Delta y$, and $|v| \leq \Delta V_{\max }$. We choose $\Delta y=1.5 h^{-1} \mathrm{Mpc}$. This size is larger than the projected virial radii of $\sim 99$ per cent of the groups in our sample. The plane-of-the-sky projection of this region is shown as an orange rectangle in Fig. 1.

(iii) We compute the galaxy overdensity in the filament region. To do so, we construct a random galaxy catalogue based on the MGS galaxies after excluding group galaxies. The random catalogue is 100 times denser, has the same redshift distribution and the same angular coverage than the MGS galaxies. We further require that these random points do not lie within the cylindrical volumes centred on groups that were used to clean up the MGS from galaxies in groups (see point (i) above). With this restriction, the volume filled by the random sample mimics that of the real data we use to identify filaments. From all group pairs we select those having a number overdensity in the filament region $\delta n / n_{r}=\left(n-n_{r}\right) / n_{r}>1$, where $n$ and $n_{r}$ are the number of MGS galaxies and the normalized number of random points in the filament region, respectively. That is, we consider that a group pair is linked with a filament if the galaxy number density in the filament region is at least twice the mean density at the pair's redshift. In Fig. 2, we show the distribution of $n / n_{r}$ of the group pairs, where the value $n / n_{r}=2$ corresponds to our choice for defining filaments. With this cut-off, out of our original sample of 3094 pairs, 2366 pairs hold filaments. It is worth noticing that most group pairs ( $\sim 76$ per cent $)$ meet our overdensity condition and thus, most group pairs should be linked by actual filaments.

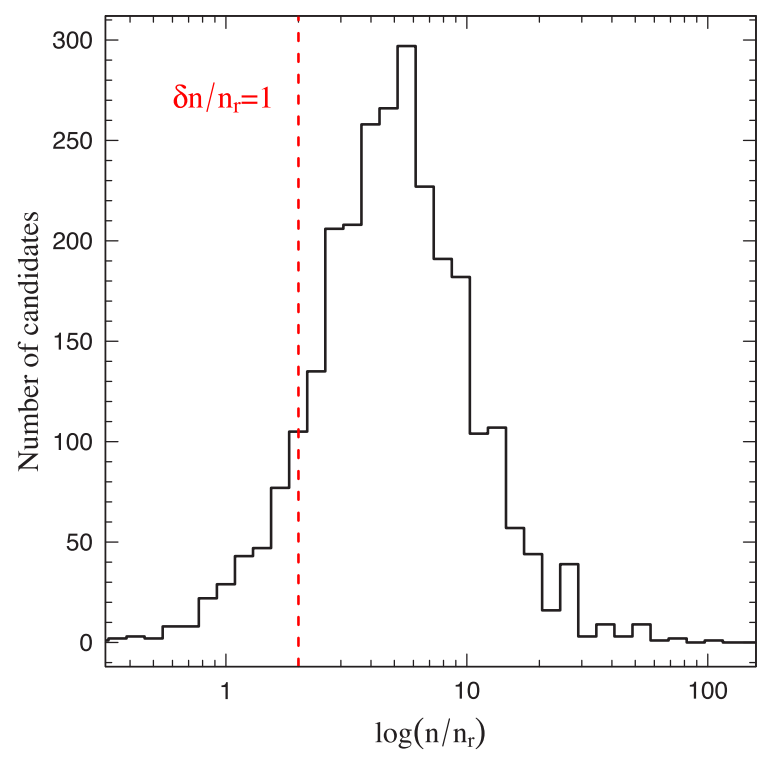

Figure 2. The distribution of the galaxy number density (relative to the random number density) in the filament region of our sample of group pairs. The vertical red dashed line corresponds to a galaxy overdensity $\delta n / n_{r}=1$, which we use to define our sample of filaments.

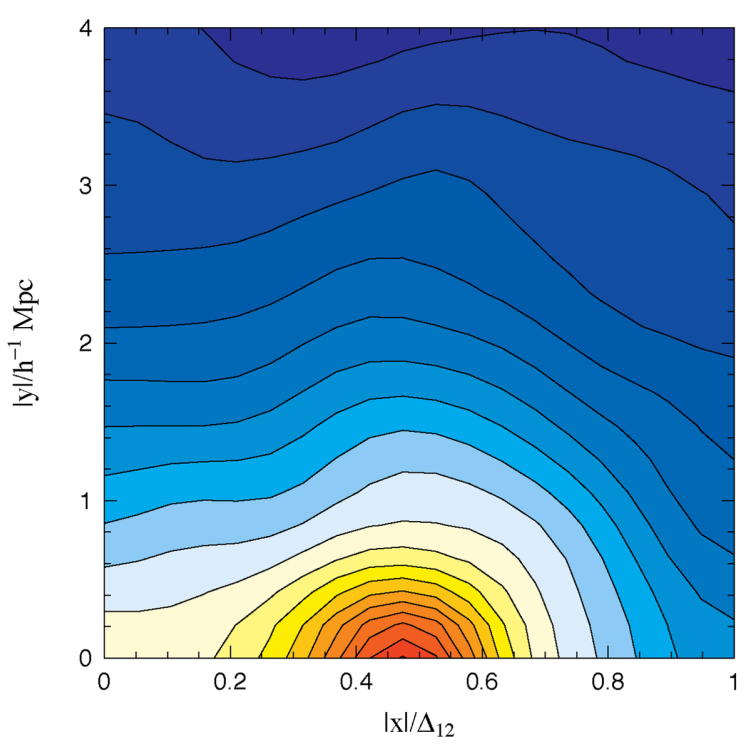

Figure 3. The projected galaxy overdensity around the stacked sample of groups with filaments. In these coordinate axes groups are centred at $(0.5,0)$ and the filament region extends from there to the origin. See text for details.

We show in Fig. 3 the galaxy overdensity in the plane of the sky for our stacked sample of group pairs with filaments as a function of $|x| / \Delta_{12}$ and $|y|$. In this figure, groups are located in the position $(0.5,0)$. Galaxies in groups have been removed. Besides the expected increase in density around the location of the groups, it is also clear the presence of an overdensity stretching from the groups towards the geometric centre of the group pairs, i.e. from $|x| / \Delta_{12}=0.5$ to $|x| / \Delta_{12}=0$. This contrasts with the circular-like behaviour of the overdensity contours in the opposite direction. 


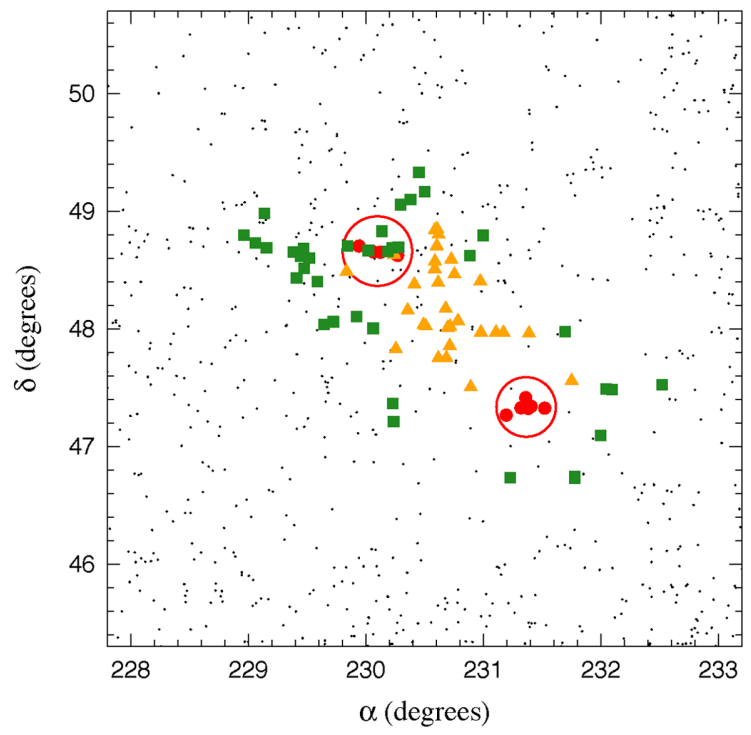

Figure 4. An example of two groups of galaxies in our sample and the galaxies we identify as infalling into them. Each group is marked by a circle which represents its projected virial radius. Galaxies belonging to each group are shown as filled red circles. Isotropically infalling galaxies are shown as green squares, while orange triangles represent galaxies in the filament region. Field galaxies are shown as black dots.

\subsection{Infalling regions around groups: filaments and isotropic infall}

We are particularly interested in exploring possible differences between galaxies infalling along the preferred directions defined by the filaments, and galaxies infalling from other directions, that we will consider to be infalling isotropically. Hereafter, we will refer to the former as FG and the latter as IG.

For each group pair we consider the FGs as falling to/associated with the closest group in projection. Every galaxy in the FG and IG samples will be considered as falling to/associated with its closest group in projection. Thus, by construction, each group in a pair contributes to the sample of FG with galaxies that can be separated as far as $\sim \Delta_{12} / 2$ in projection. We use that distance to define the isotropic infall region around each group: a cylinder centred in the group and oriented in the line of sight direction defined by a radius $\Delta_{12} / 2$ and a height $2 \Delta V_{\max }$. We show the projection in the sky of these cylinders as green dashed circles in Fig. 1. Each group contributes to the IG sample up to the same scale as it does to the sample of galaxies in filaments. An example of an actual group pair along with the galaxies in its filament region and in the isotropic regions, can be seen in Fig. 4. The samples of IG and FG comprise 33094 and 26043 galaxies, respectively. According to the criteria described above, a galaxy cannot be classified simultaneously as both: FG and IG. Nevertheless, it is worth mentioning that an FG can be a member of more than one filament.

Our samples of IG and FG include galaxies that are effectively in the isotropic infalling region or in the filaments, respectively. It is clear that, both samples will be contaminated by foreground and background galaxies, and backsplash galaxies. Unless we had three dimensional positions and velocities, we are unable to isolate the actual IG and FG samples. By construction, the IG and FG samples have similar redshift distributions, and we expect both to be contaminated with foreground and background in the same way. Therefore, any difference in the galaxy properties of the samples should reflect an actual and more significant difference in the populations.

\subsection{Control samples: field and group galaxies}

To understand the effects of the infall regions on galaxies, an adequate comparison with samples of galaxies in the field and in groups is needed.

We construct a sample of field galaxies drawn from the MGS DR7 by randomly selecting galaxies in the redshift range under consideration, avoiding in the process all MZ11 groups, filaments and isotropic infall regions. We impose to this sample of field galaxies to have the same redshift distribution as the FG and IG samples. Our resulting sample of field galaxies comprises 156357 galaxies.

The sample of galaxies in groups contains all galaxies in the groups with filaments, adding up a total of 19464 galaxies.

\section{COMPARING GALAXY POPULATIONS IN THE INFALLING REGIONS, FIELD AND GROUPS}

In this section, we perform a comparison of properties of the galaxy population inhabiting filaments, isotropic infall region, field, and groups. We focus our analyses on the luminosity and the star formation of galaxies.

\subsection{Luminosity function}

We use two methods to compute the ${ }^{0.1} r$-band LF of galaxies in our samples: the non-parametric $C^{-}$(Lynden-Bell 1971; Choloniewski 1987) for the binned LF, and the STY method (Sandage, Tammann \& Yahil 1979) to compute the best-fitting Schechter (1976) function parameters: the faint-end slope $\alpha$, and the characteristic absolute magnitude $M^{*}$. We also compute separately the LF of early and late type galaxies according to their concentration parameter Strateva et al. (2001). In Fig. 5, we show the resulting binned LFs in arbitrary

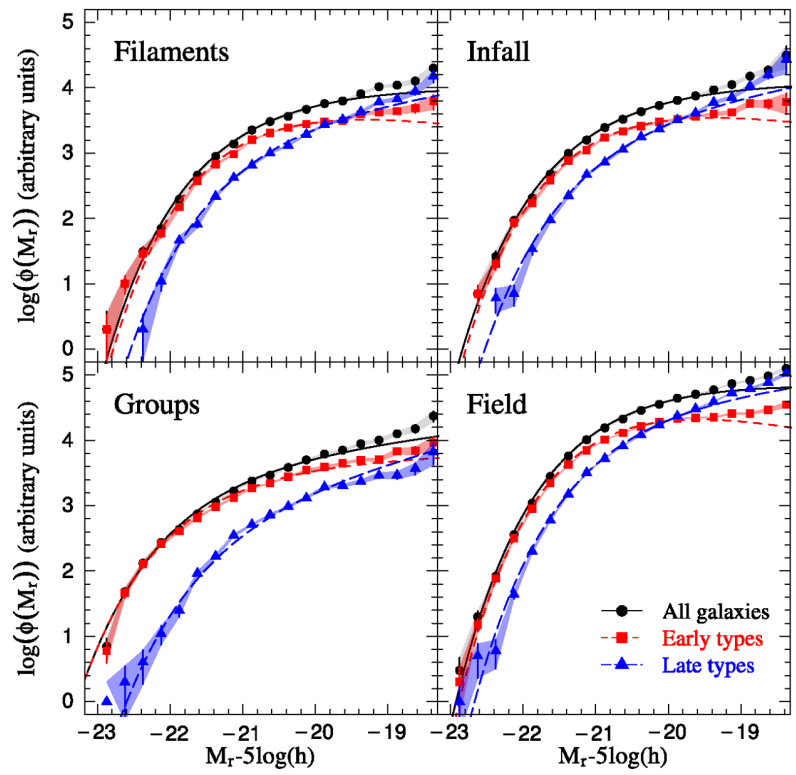

Figure 5. The ${ }^{0.1} r$-band luminosity functions of galaxies in the four different environments we probe: Filaments, isotropic infall area, groups and field. We show in filled black circles the LF of all galaxies irrespective of their type; red squares correspond to early-type galaxies, and blue triangles to latetype galaxies. Points were calculated using the $C^{-}$method and error-bars using the bootstrap resampling technique. Best-fitting Schechter functions were computed using the STY method. Best-fitting parameters are shown in Fig. 6 below. 


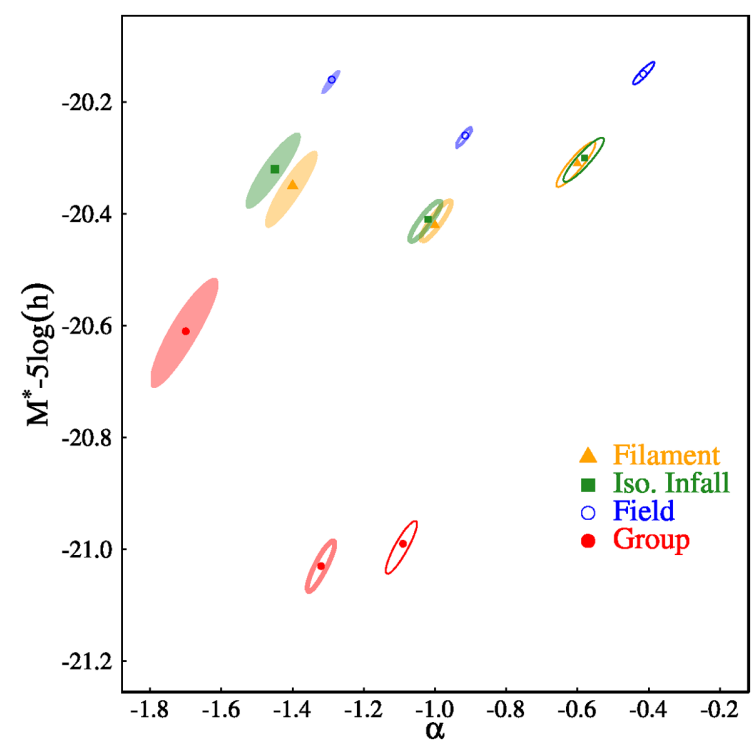

Figure 6. Best-fitting Schechter parameters of the luminosity functions shown in Fig. 5 and quoted in Table 1. Points are the best-fitting values, shown along their $1 \sigma$ contours. Different colours and symbols indicate the environment: blue open circles correspond to field galaxies; green squares to IG, orange triangles to FG, and red filled circles to galaxies in groups. Different type of contours indicate galaxy type: filled contours correspond to late types, contours enclosed by thin lines correspond to early types, and contours enclosed by thick lines correspond to all galaxies, irrespective of their type.

units, also shown in this figure are the best-fitting Schechter functions. The Schechter parameters along with their $1 \sigma$ error contours are shown in Fig. 6 separately for the complete samples and the subsamples of early and late-types. The values of the parameters are displayed in Table 1.

It is easier to spot similarities and differences between the populations by inspecting Fig. 6. As expected, and regardless of type, field and group galaxies are the two extremes cases: the former have the shallowest faint-end slope and the faintest characteristic absolute magnitude, while the latter are the opposite. In between them, the LF parameters of FGs and IGs are closer to, however different from, those of field galaxies. The LF of FG and IG are indistinguishable for the complete sample of galaxies and for early-types. A subtle difference is observed for late-types: FG has a shallower faint end

Table 1. Best-fitting Schechter's parameters of the luminosity functions shown in Fig. 5 computed through the STY method. See also Fig. 6.

\begin{tabular}{lccc}
\hline Environment & Galaxy type & $\alpha$ & $\begin{array}{c}M^{*}-5 \log (h) \\
0.1\end{array}$ \\
& & & $-20.26 \pm 0.02$ \\
Field & All & $-0.91 \pm 0.02$ & $-20.15 \pm 0.01$ \\
& Early type & $-0.41 \pm 0.02$ & $-20.016 \pm 0.02$ \\
& Late type & $-1.29 \pm 0.02$ & -20.16 .040 .04 \\
Isotropic & All & $-1.02 \pm 0.05$ & $-20.41 \pm 0.06$ \\
Infall & Early type & $-0.58 \pm 0.06$ & $-20.30 \pm 0.04$ \\
& Late type & $-1.45 \pm 0.07$ & $-20.32 \pm 0.07$ \\
Filament & All & $-1.00 \pm 0.05$ & $-20.42 \pm 0.04$ \\
& Early type & $-0.60 \pm 0.06$ & $-20.31 \pm 0.04$ \\
& Late type & $-1.40 \pm 0.07$ & $-20.35 \pm 0.07$ \\
Groups & All & $-1.32 \pm 0.04$ & $-21.03 \pm 0.05$ \\
& Early type & $-1.09 \pm 0.04$ & $-20.99 \pm 0.05$ \\
& Late type & $-1.70 \pm 0.09$ & $-20.6 \pm 0.1$ \\
\hline
\end{tabular}

slope, and its characteristic magnitude is brighter. This is, however, only one sigma difference. It is worth noticing that the values of $\alpha$ and $M^{*}$ for our sample of galaxies in groups, regardless of galaxy type, are fully consistent with the results by ZM11 for groups with masses in the range of our sample. A straightforward comparison for field galaxies cannot be made with previous determinations of the LF of galaxies (e.g. Montero-Dorta \& Prada 2009), given our particular definition of field galaxies, which excludes galaxies in groups and in the infalling regions of groups.

\subsection{Specific star formation rate}

We search now for differences in the star formation of the galaxies in our samples. In particular, we focus our attention on the specific star formation rate, and its dependence with stellar mass and the distance to the nodes of the filaments.

The values of stellar mass and SSFR for the galaxies in our samples have been extracted from the MPA-JHU DR7 release of spectra measurements. ${ }^{1}$ This catalogue provides, among other parameters, stellar masses based on fits to the photometry following Kauffmann et al. (2003) and Salim et al. (2007), and star formation rates based on Brinchmann et al. (2004).

In Fig. 7, we show the fraction of low SSFR $\left(\log \left(\mathrm{SSFR} / \mathrm{yr}^{-1}\right)\right.$ $<-11)$ as a function of stellar mass for our samples of galaxies. Lowest mass bins are numerically dominated by late-types and the highest mass bins by early-types. Regardless of whether we consider all galaxies, or whether we split them into early and late types, group and field galaxies exhibit the extreme values: over the stellar mass range we probe, groups have the highest fraction of low-SSFR galaxies, while the opposite occurs for field galaxies. In between them, but typically closer to field values are FG and IG. Filaments have systematically a fraction of low-SSFR galaxies higher than the isotropic infall region, for stellar masses higher than $\sim 10^{10} \mathrm{M}_{\odot}$.

To check whether these differences between FG and IG are indicative of actual differences between the populations we rely on the test used by Muriel \& Coenda (2014). Briefly, let us consider two samples of objects, $A$ and $B$, and two physical quantities $X$ and $Y$. This test allows us to tell whether the two populations have different trends of $Y$ as a function of $X$. Let us consider $N_{\text {bin }}$ bins of the $X$ variable, and let $N_{\mathrm{A}}^{(i)}$ and $N_{\mathrm{B}}^{(i)}$ be the numbers of objects of the samples $A$ and $B$ in the $i$ th bin, respectively. Now let us consider, within each bin, the sum of the differences in the quantity $Y$ from all the pairs formed by one object from the sample $A$ and the other from the sample $B$. After accumulating over all bins and normalizing by the number of pairs used in the process, we arrive to the quantity:

$\Delta_{\text {obs }} \equiv \frac{\sum_{i=1}^{N_{\text {bin }}} \sum_{j=1}^{N_{\mathrm{A}}^{(i)}} \sum_{k=1}^{N_{\mathrm{B}}^{(i)}}\left(Y_{\mathrm{A}, \mathrm{j}}^{(i)}-Y_{\mathrm{B}, \mathrm{k}}^{(i)}\right)}{\sum_{l=1}^{N_{\text {bin }}} N_{\mathrm{A}}^{(l)} N_{\mathrm{B}}^{(l)}}$,

where $Y_{\mathrm{A}, \mathrm{j}}^{(i)}$ and $Y_{\mathrm{B}, \mathrm{k}}^{(i)}$ are the $Y$ values of the $j$ th object of the sample $A$ and the $k$ th object of the sample $B$ in the $i$ th bin, respectively. With this definition, $\Delta_{\text {obs }}$ is a measure of the average difference in the quantity $Y$ between the two samples, once the overall trend of $Y$ as a function of $X$ has been removed. If the two samples had no differences regarding the behaviour of $Y$ as a function of $X$, then we would expect $\Delta_{\mathrm{obs}} \simeq 0$. On the other hand, a non-zero value cannot be straightforwardly interpreted as mirroring a significant difference between the samples, unless the obtained value is unlikely for the

\footnotetext{
${ }^{1}$ http://www.mpa-garching.mpg.de/SDSS/DR7
} 

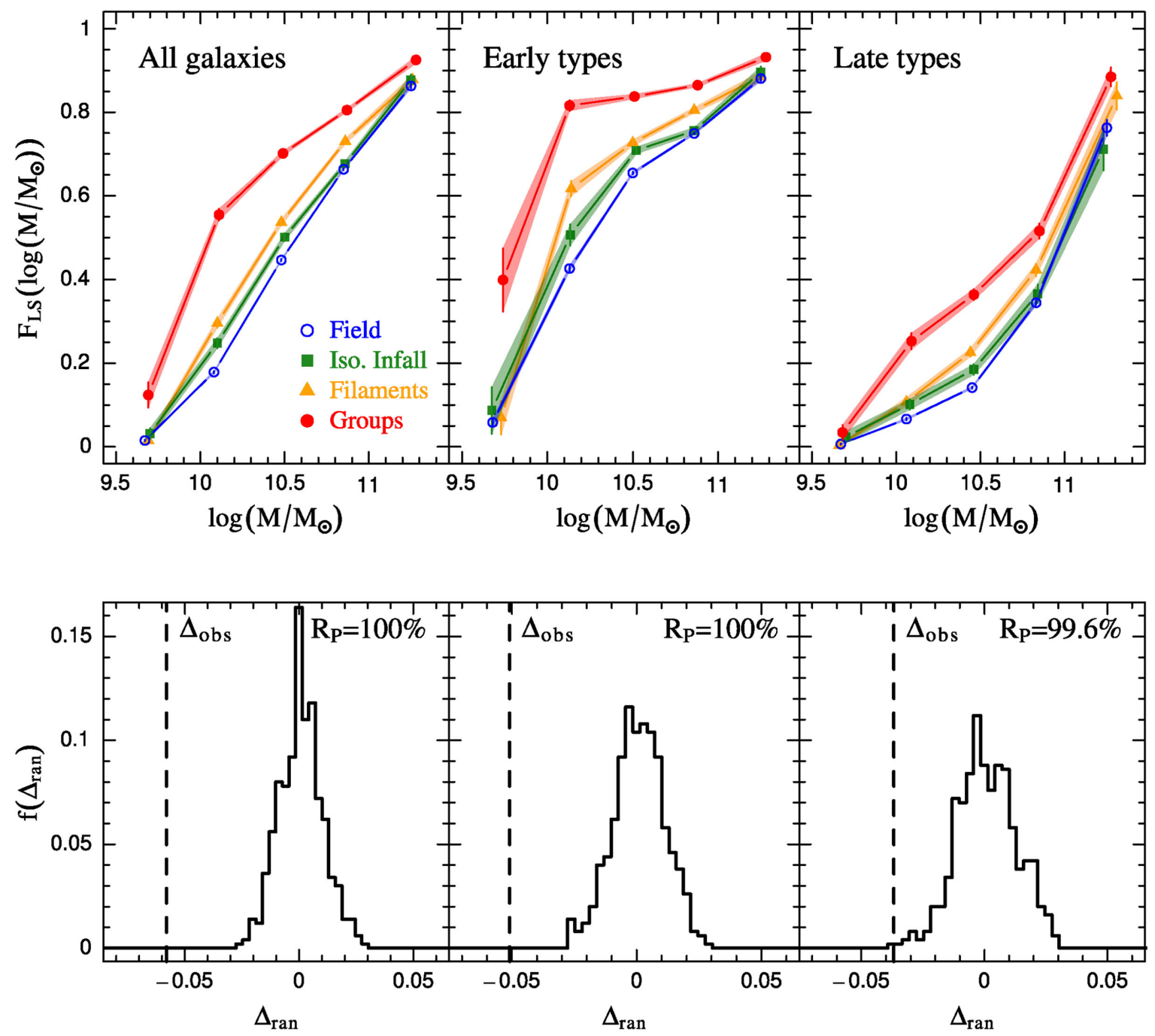

Figure 7. The dependence of the SSFR on stellar mass. Upper panels: the fraction of low-SSFR $\left(\log \left(\mathrm{SSFR} / \mathrm{yr}^{-1}\right)<-11\right) \mathrm{galaxies}, F_{\mathrm{LS}}$, as a function of stellar mass. Left-hand panel includes all galaxies, while the centre and right-hand panels consider only early and late types, respectively. Error-bars were computed using the bootstrap resampling technique. In the lower panels, we show the results of applying the test of Section 3.2 for the null hypothesis that there are no differences in the SSFR as a function of stellar mass between the IG and FG samples of the upper panels. Each panel shows the normalized distributions of the quantity $\Delta_{\text {ran }}$, the values of $\Delta_{\text {obs }}$ (vertical dashed lines), and the rejection probability, $R_{\mathrm{P}}$, of the null hypothesis (see Table 2).

null hypothesis corresponding to the case in which both samples are drawn from the same underlying population.

Let $C$ be the sample resulting by the merging of samples $A$ and $B$. By construction, this sample has $N_{\mathrm{C}}^{(i)}=N_{\mathrm{A}}^{(i)}+N_{\mathrm{B}}^{(i)}$ in the $i$ th bin. Now, let us randomly select from $C$ two subsamples $A^{\prime}$ and $B^{\prime}$, bound to have the same number of objects per bin as the samples $A$ and $B$, respectively. Clearly, each of these new samples will include objects from both, $A$ and $B$. From the samples $A^{\prime}$ and $B^{\prime}$ we compute the value $\Delta_{\text {ran }}$. If we repeat this procedure a large number of times, performing a different random selection each time, $\Delta_{\text {ran }}$ will be distributed around the value 0 . Provided $\Delta_{\text {obs }}>0$, we can now quantify the rejection probability of the null hypothesis by computing the fraction, $F$, of random realizations that give $\Delta_{\text {ran }}>$ $\Delta_{\text {obs }}$ : the rejection probability will be $R_{\mathrm{P}}=1-F$. In the case , $\Delta_{\text {obs }}$ $<0, F$ is defined as the fraction of random realizations that give $\Delta_{\text {ran }}<\Delta_{\text {obs }}$. The sign of $\Delta_{\text {obs }}$ tell us which of the samples has systematically larger values of the quantity $Y$ as a function of $X$.

Results of 1000 random realizations of the test can be seen in the bottom panels of Fig. 7 and in Table 2, where we use $X=\log (M)$ and
Table 2. The dependence of SSFR on the stellar mass: results of applying the test of Section 3.2 to the samples of galaxies in the isotropic infall region and the sample of galaxies in filaments. See also Fig. 7.

\begin{tabular}{lcc}
\hline Galaxy type & $\begin{array}{c}\Delta_{\text {obs }} \\
\left(\log \left(\mathrm{SSFR} / \mathrm{yr}^{-1}\right)\right)\end{array}$ & $\begin{array}{c}\text { Rejection } \\
\text { probability }\end{array}$ \\
\hline All & 0.079 & 100 per cent \\
Early types & 0.069 & 100 per cent \\
Late types & 0.040 & 99.9 per cent \\
\hline
\end{tabular}

$Y=$ SSFR. The test is conclusive: irrespective of galaxy type, the null hypothesis of FG and IG being drawn from the same underlying population is ruled out at a confidence level above 99 percent in the three cases. This means that FG and IG have significant different trends in their SSFR as a function of stellar mass. Besides the surrounding large-scale structure, galaxies can be affected by their small-scale local environment. Evidence has been found that galaxies in pairs have their star formation 


\section{All galaxies $\quad$ Early types $\quad$ Late types}

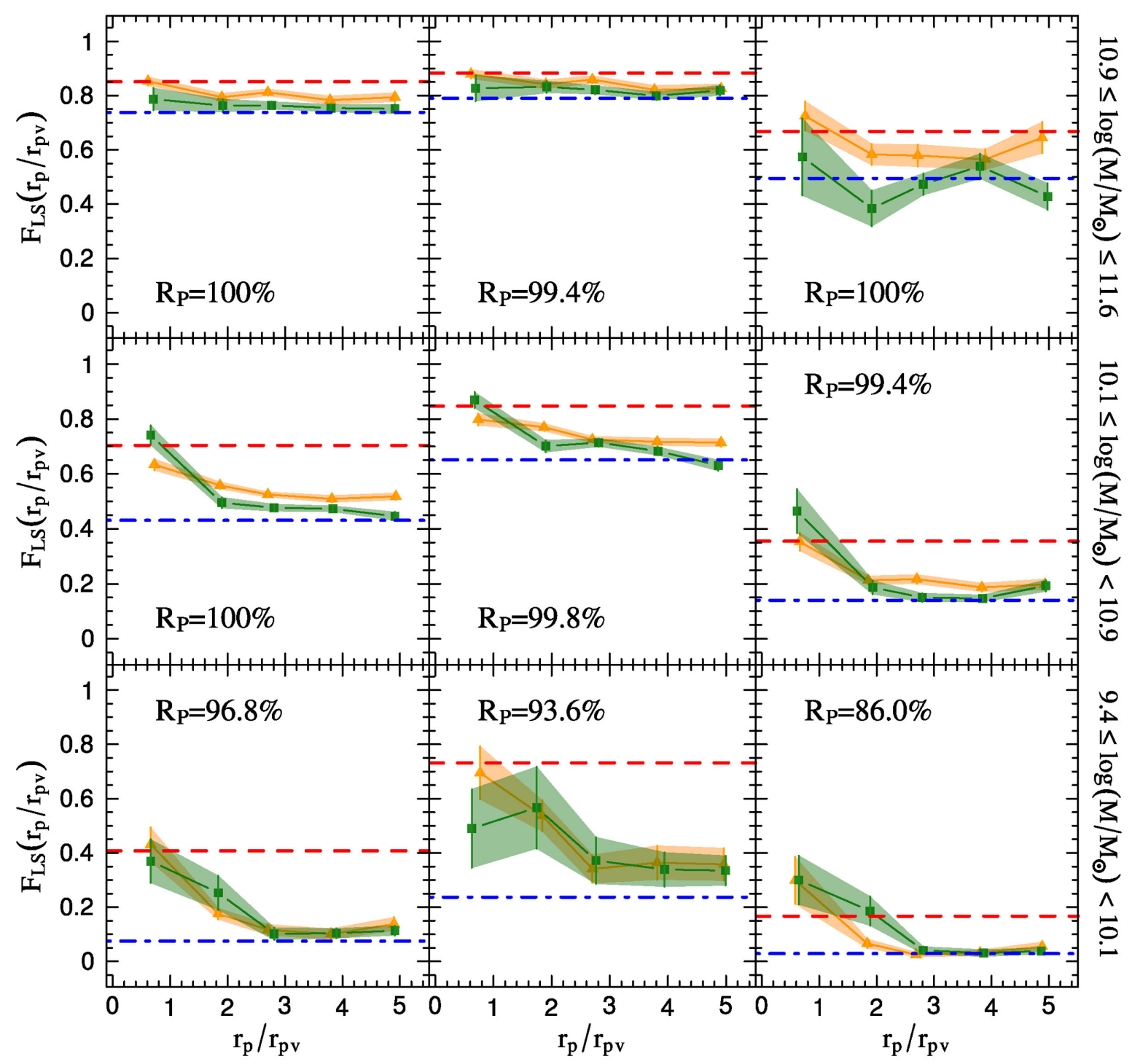

Figure 8. The fraction of low-SSFR $\left(\log \left(\mathrm{SSFR} / \mathrm{yr}^{-1}\right)<-11\right)$ galaxies, $F_{\mathrm{LS}}$, as a function of the projected distance to the nearest node, $r_{\mathrm{p}}$, in units of the projected virial radius of the nearest node, $r_{\mathrm{pv}}$. Left column: all galaxies, central column: early-type galaxies, and right column: late-type galaxies. Rows correspond to the mass intervals quoted in the right side of the figure. Galaxy mass decreases from top to bottom. Green squares represent IG and orange triangles FG. Error-bars were computed using the bootstrap resampling technique. Horizontal red long-dashed lines, and blue dashed-dotted lines are the fraction of low-SSFR galaxies in groups and in the field, respectively. We quote inside each panel the rejection probability, $R_{\mathrm{P}}$, of the null hypothesis that there are no differences in the SSFR as a function of projected distance between the IG and FG samples (see Table 3).

suppressed by each other, see for instance Alpaslan et al. (2015). To test whether our results could be due to a relative excess of pairs in the FG sample, we compute the fraction of galaxies in the FG and IG samples that are in pairs. For each galaxy in these samples, we search for a pair in the MGS, using the criterion by Alpaslan et al. (2015). We find that there are no significant differences between the fraction of galaxies in pairs in FG (7.3 per cent) and in IG (7.1 per cent).

Our results so far, show that both, FG and IG are different from field galaxies (and group galaxies) in terms of their SSFR. For a galaxy, the fact of being close to a massive group modifies its star formation rate. Even more, how a galaxy's star formation is affected depends on whether it is located in a filament or in the isotropic infall zone. In what follows, we analyse how the impact of the environment on star formation depends on distance to the nodes.

In Fig. 8, we show the fraction of low-SSFR galaxies, as a function of the projected distance to the nearest node in units of the node's projected virial radius. Since SSFR depends on stellar mass, we split our samples into three bins of stellar mass (rows in Fig. 8), and also analyse separately each galaxy type (columns in Fig. 8). We also show in this figure, the mean values corresponding to field and group galaxies as dashed horizontal lines. As a general trend, the fraction of low-SSFR galaxies rises towards the nodes, and smoothly decreases outwards. We find that:

(i) for massive galaxies, which are dominated in number by earlytypes, the dependence of the fraction of low SSFR on distance, for 
Table 3. The dependence of SSFR on the projected distance to the nodes. Results of applying the significance test of Section 3.2 to the samples of galaxies in filaments and in the isotropic infall region (Fig. 8).

\begin{tabular}{lccc}
\hline Galaxy type & $\begin{array}{c}\text { Stellar mass range } \\
\left(\log \left(\mathrm{M} / \mathrm{M}_{\odot}\right)\right)\end{array}$ & $\begin{array}{c}\Delta_{\text {obs }} \\
\left(\log \left(\mathrm{SSFR}_{\mathrm{yr}}{ }^{-1}\right)\right)\end{array}$ & $\begin{array}{c}\text { Rejection } \\
\text { probability }\end{array}$ \\
\hline All & $9.40-10.13$ & 0.157 & 96.8 per cent \\
Early types & $9.40-10.13$ & 0.728 & 93.6 per cent \\
Late types & $9.40-10.13$ & 0.019 & 86.0 per cent \\
All & $10.13-10.87$ & 0.055 & 100 per cent \\
Early types & $10.13-10.87$ & 0.041 & 99.8 per cent \\
Late types & $10.13-10.87$ & 0.035 & 99.4 per cent \\
All & $10.87-11.60$ & 0.047 & 100 per cent \\
Early types & $10.87-11.60$ & 0.033 & 99.4 per cent \\
Late types & $10.87-11.60$ & 0.133 & 100 per cent \\
\hline
\end{tabular}

both FG and IG, is quite flat. FG have a higher fraction of low-SSFR galaxies over the whole range. The fraction of low-SSFR IG is either, consistent with, or marginally larger than the field value. In any case, no differences with the field are observed beyond $\sim 3 r_{\mathrm{pv}}$. On the other hand, FG do not reach field values, even though their lowSSFR fraction decreases with distance. The rejection probability, now using $\mathrm{X}=r / r_{\mathrm{pv}}$, in all cases indicates that the differences between IG and FG are significant (see Table 3). For late types IG, it is interesting to note the data point at $r_{\mathrm{p}} \sim 2 r_{\mathrm{pv}}$, which is a $\sim 2 \sigma$ signal indication of an enhancement in the star formation. A similar effect has been reported for galaxies infalling into clusters by Porter et al. (2008) and Mahajan et al. (2012).

(ii) For intermediate mass galaxies, the effects of the filamentary environment on the star formation are stronger. FG do not reach field values in the whole range probed. IG are marginally more affected in scales $r_{\mathrm{p}} \leq 1.4 r_{\mathrm{pv}}$. Again, the rejection probability in all cases means that differences between IG and FG are significant.

(iii) For low-mass galaxies, numerically dominated by late-types, no significant differences between FG and IG are seen, this is mirrored by the low values of the rejection probability. No significant departures from field values are observed beyond $\sim 3 r_{\mathrm{pv}}$ for the whole sample and for late types. However, for early types, field values are not reached at all over the whole range explored.

\section{DISCUSSION AND CONCLUSIONS}

In this paper, we study the effect of environment upon galaxies infalling into groups. For this purpose, we search for filamentary structures connecting massive groups of galaxies using samples of groups and galaxies taken from the SDSS DR7. We compare properties of galaxies around these filaments' nodes, selected according to their projected distances and their radial velocity difference. We classify galaxies in the vicinity of these groups in two cases: those that are in the filament region (FG), and those that are in the isotropic infall region (IG). It is clear that both of these samples suffer from contamination from foreground and background galaxies. The use of control samples of field, and group galaxies, allows us to spot the actual differences between infalling galaxies and galaxies in the field and in groups.

Our comparison focuses in two physical properties of galaxies: luminosity, by means of the analysis of the luminosity functions; and, specific star formation rate, by studying its dependence on stellar mass, galaxy type, and projected distance to the nodes.

We find that the luminosity functions of FG and IG galaxies are basically indistinguishable between them, with the possible exception of late-types. On the other hand, and regardless of type, both of them are clearly different from those of field or group galaxies. Galaxies in filaments and in the isotropic infall region have characteristic absolute magnitudes brighter by $\sim 0.2$ magnitudes when compared to field galaxies, and fainter by $\sim 0.6$ magnitudes compared to galaxies in the nodes. These differences are larger when we consider early types, and smaller when we consider late types. A similar effect is seen in the faint end slope: it is larger (in absolute values) in $\sim 0.1$ for LG and IG when compared to the field, and smaller in $\sim 0.3$ when compared to the nodes' value. Again, differences are larger for early types, and smaller for late types. Due to the apparent magnitude limit of the main galaxy sample of SDSS, we are not probing faint magnitudes, and thus, the faint end slope of the LF is basically computed from the convexity of the LF around the characteristic magnitude. It is clear that, regarding the luminosity, FG and IG differ from both: field galaxies and galaxies in the nodes.

Significant differences between FG and IG appear when we analyse the SSFR. Regardless of type, all samples analysed here, have different trends of SSFR as a function of stellar mass. The samples of field and group galaxies are the two extremes, with the lowest and highest fraction of low-SSFR galaxies, respectively. In between them, FG have a significantly larger fraction of low-SSFR galaxies than IG. Thus, not only these two samples differ from the field and the groups, but also they have been affected differently by the environment. Clearly, galaxies infalling into groups along filamentary structures have experienced a stronger quenching in their star formation than galaxies infalling into groups from other directions.

Another clear indication that filamentary structures have a distinct impact on galaxy evolution appears when we analyse the dependence of the SSFR with the projected distance to the nodes of the filaments. We find that the fraction of low-SSFR galaxies increases towards the nodes and decreases outwards for both, IG and FG. However, FG have a typically larger fraction of low-SSFR galaxies compared to IG up to $\sim 3 r_{\text {pv }}$ projected radii. These differences are better seen for high and intermediate mass galaxies. Our results also show that filaments affect star formation further out than the isotropic infalling region does. Over the range of distances we probe, we do not find any enhancement of the star formation relative to the field, with the exception of massive late types IG. These galaxies have an excess of high-SSFR galaxies compared to the field at a projected distance of $r_{\mathrm{p}} \sim 2 r_{\mathrm{pv}}$. This may be consistent with the findings of Porter et al. (2008) and Mahajan et al. (2012) in the outskirts of clusters.

Our results show that galaxies infalling into massive groups differ from field galaxies regarding their star formation up to $\sim 3 r_{\mathrm{pv}}$, and even further out if they are located in filaments. Galaxies infalling into groups have lower star formation than field galaxies, but still not as low as group galaxies. This quenching of star formation is stronger in filaments. On the other hand, both, FG and IG are already typically brighter than field counterparts, still not as much as group galaxies. These two results are an indication that some of the physical mechanisms that determine the differences observed between field galaxies and galaxies in systems, affect galaxies even when they are not yet within the systems.

\section{ACKNOWLEDGEMENTS}

We thank the referee, Mehmet Alpaslan, for his comments which improved the paper. This work was supported with grants from CONICET (PIP 11220120100492CO and 11220130100365CO) and SECYT-UNC, Argentina. Funding for the Sloan Digital Sky Survey (SDSS) has been provided by the Alfred P. Sloan 
Foundation, the Participating Institutions, the National Aeronautics and Space Administration, the National Science Foundation, the U.S. Department of Energy, the Japanese Monbukagakusho, and the Max Planck Society. The SDSS Web site is http://www.sdss.org/. The SDSS is managed by the Astrophysical Research Consortium for the Participating Institutions. The Participating Institutions are The University of Chicago, Fermilab, the Institute for Advanced Study, the Japan Participation Group, The Johns Hopkins University, the Korean Scientist Group, Los Alamos National Laboratory, the Max Planck Institut für Astronomie, the Max Planck Institut für Astrophysik, New Mexico State University, University of Pittsburgh, University of Portsmouth, Princeton University, the United States Naval Observatory, and the University of Washington.

\section{REFERENCES}

Abazajian K. N. et al., 2009, ApJS, 182, 543

Aihara H. et al., 2011, ApJS, 193, 29

Alpaslan M. et al., 2014, MNRAS, 438, 177

Alpaslan M. et al., 2015, MNRAS, 451, 3249

Aragón-Calvo M. A., van de Weygaert R., Jones B. J. T., 2010, MNRAS, 408, 2163

Beers T. C., Flynn K., Gebhardt K., 1990, AJ, 100, 32

Blanton M. R. et al., 2003, AJ, 125, 2348

Blanton M. R. et al., 2003a, AJ, 125, 2348

Bond J. R., Kofman L., Pogosyan D., 1996, Nature, 380, 603

Bond N. A., Strauss M. A., Cen R., 2010, MNRAS, 409, 156

Brinchmann J., Charlot S., White S. D. M., Tremonti C., Kauffmann G., Heckman T., Brinkmann J., 2004, MNRAS, 351, 1151

Choloniewski J., 1987, MNRAS, 226, 273

Colberg J. M., Krughoff K. S., Connolly A. J., 2005, MNRAS, 359, 272

Ebeling H., Barrett E., Donovan D., 2004, ApJ, 609, L49

Ellingson E., Lin H., Yee H. K. C., Carlberg R. G., 2001, ApJ, 547, 609

González R. E., Padilla N. D., 2010, MNRAS, 407, 1449

Guo Q., Tempel E., Libeskind N. I., 2015, ApJ, 800, 112
Huchra J. P., Geller M. J., 1982, ApJ, 257, 423

Just D. W. et al., 2015, preprint (arXiv:1506.02051)

Kauffmann G. et al., 2003, MNRAS, 341, 33

Limber D. N., Mathews W. G., 1960, ApJ, 132, 286

Lynden-Bell D., 1971, MNRAS, 155, 95

Mahajan S., Raychaudhury S., Pimbblet K. A., 2012, MNRAS, 427, 1252

Montero-Dorta A. D., Prada F., 2009, MNRAS, 399, 1106

Moran S. M., Ellis R. S., Treu T., Smith G. P., Rich R. M., Smail I., 2007, ApJ, 671, 1503

Muriel H., Coenda V., 2014, A\&A, 564, A85

Patel S. G., Kelson D. D., Holden B. P., Franx M., Illingworth G. D., 2011, ApJ, 735, 53

Pimbblet K. A., 2005, MNRAS, 358, 256

Pimbblet K. A., Drinkwater M. J., Hawkrigg M. C., 2004, MNRAS, 354, L61

Porter S. C., Raychaudhury S., Pimbblet K. A., Drinkwater M. J., 2008, MNRAS, 388, 1152

Rines K., Geller M. J., Kurtz M. J., Diaferio A., 2005, AJ, 130, 1482

Salim S. et al., 2007, ApJS, 173, 267

Sandage A., Tammann G. A., Yahil A., 1979, ApJ, 352, 82

Schechter P., 1976, ApJ, 203, 297

Schlegel D. J., Finkbeiner D. P., Davis M., 1998, ApJ, 500, 525

Smith A. G., Hopkins A. M., Hunstead R. W., Pimbblet K. A., 2012, MNRAS, 422, 25

Stoica R. S., Martínez V. J., Saar E., 2010, A\&A, 510, A38

Strateva I. et al., 2001, AJ, 122, 1861

Strauss M. A. et al., 2002, AJ, 124, 1810

Verdugo M., Ziegler B. L., Gerken B., 2008, A\&A, 486, 9

York D. G. et al., 2000, AJ, 120, 1579

Zandivarez A., Martínez H. J., 2011, MNRAS, 415, 2553 (ZM11)

Zandivarez A., Merchán M. E., Padilla N. D., 2003, MNRAS, 344, 247

Zhang Y., Dietrich J. P., McKay T. A., Sheldon E. S., Nguyen A. T. Q., 2013, ApJ, 773, 115

This paper has been typeset from a $\mathrm{T}_{\mathrm{E}} \mathrm{X} / \mathrm{LT} \mathrm{T} \mathrm{X}$ file prepared by the author. 